\title{
Los programas de fidelización como plataforma de venta directa: clasificación de los titulares según la redención de billetes aéreos
}

\author{
Loyalty programmes as a direct sales platform: \\ a cardholder segmentation based on air flight \\ redemptions
}

\author{
José Manuel Ponzoa Casado ${ }^{1}$ \\ Pedro Reinares Lara ${ }^{2}$
}

Recibido el 27 de enero de 2010, aceptado el 8 de julio de 2010

$\mathrm{N}^{\mathrm{o}}$ de clasificación JEL: M31

DOI: $10.5295 /$ cdg. 100206jp

Reseña bibliográfica: PONZOA, J. y REINARES, P. (2010): "Los programas de fidelización como plataforma de venta directa: clasificación de los titulares según la redención de billetes aéreos", Cuadernos de Gestión, Vol 10, nº especial, pp. 197-213, DOI: 10.5295/cdg.100206jp

\section{Resumen:}

Cada vez son más numerosos los programas de fidelización que ofrecen al titular la posibilidad de comprar puntos o conseguir premios, viajes o billetes aéreos pagando una parte de los mismos con dinero. Dicha característica, unida a la propia estructura y dinámica de los programas de fidelización y a la actual coyuntura del sector turístico, ha permitido desarrollar plataformas de venta directa desde las que ofrecer servicios a los titulares.

Este trabajo argumenta el potencial de los programas de fidelización como instrumento para el conocimiento y la segmentación de clientes, diferenciando entre titulares que canjean premios con cargo exclusivamente a puntos y aquellos que lo hacen con puntos más dinero.

Considerando un programa de fidelización multisponsor líder en España, el artículo analiza la importancia del canje de billetes aéreos respecto a otras recompensas y la aportación de dinero como vía para completar el canje. Se utiliza como método de clasificación árboles de decisión para determinar cómo las variables asociadas al comportamiento de compra en las empresas asociadas a los programas influyen en el canje de billetes aéreos por parte de sus titulares.

\section{Abstract:}

There are increasingly more loyalty programmes that offer cardholders the opportunity of buying additional programme points or obtaining rewards, such as package holidays or air flights, by paying some of the cost in

\footnotetext{
${ }^{1}$ Universidad Complutense de Madrid. Facultad de CC. Económicas y Empresariales. Departamento de Comercialización e Investigación de Mercados. Campus de Somosaguas. Pabellón 6 (Las Caracolas). 28223 Pozuelo de Alarcón (MADRID).jmponzoa@ccee.ucm.es

${ }^{2}$ Universidad Rey Juan Carlos. Facultad de Ciencias Jurídicas y Sociales. Departamento de Economía de la Empresa. Campus de Vicálvaro. Paseo Artilleros s/n. 28032 Madrid. pedro.reinares@urjc.es
} 
Los programas de fidelización como plataforma de venta directa: clasificación de los titulares ...

cash. This feature of the programmes, together with their very nature and structure and the current situation in which the tourism sector finds itself, has allowed for their development as sales platforms offering services directly to their members.

This work discusses the potential of such programmes as a tool for a better knowledge and segmentation of customers by differentiating between them on the basis of those who redeem their rewards on a points-only basis and those that do so using points-plus-cash.

Using information from a leading, Spanish, multi-sponsor, loyalty programme, this article analyses the importance of redeeming air tickets only, as against other rewards, and cash contributions as a means of completing the redemption transaction. By means of decision trees, as a segmentation method, it can be seen how variables related with buying behaviour, within the programme member companies, have an influence on the redemption of air tickets by the programme's cardholders.

\section{Palabras clave:}

Programas de Fidelización, Marketing Turístico, Árboles de decisión, Venta Directa

\section{Key Words:}

Loyalty Programmes, Tourism Services, Decision trees. Direct Selling. 


\section{INTRODUCCIÓN}

La decisión de implementar un Programa de Fidelización surge de una empresa, o grupo de empresas (en el caso de los programas Multisponsor), que se plantea como objetivo incluir a sus clientes, o a un selecto grupo de ellos, en una plataforma de interacción (en términos de intercambio económico y comunicativo) en la que serán identificados y gratificados por su participación (Yi y Jeon, 2003). Se considera que los programas de fidelización, adecuadamente gestionados, permiten acciones estructuradas y operativas para la gestión, selección, relación y control del comportamiento de compra de los clientes (Michels y Bowen 2004; Banasiewicz, 2005; Carlsson y Löfgren, 2006). Por medio de sus cualidades transaccionales y relacionales, estos programas pueden convertirse en el centro de soluciones eficaces de marketing (Meyer-Waarden y Benavent, 2006).

Precisamente el origen de los programas modernos parte de las compañías aéreas. En el año 1981, American Airlines introduce el primer programa con los elementos que actualmente son propios de estas prácticas ${ }^{3}$. Conocer cómo gestionar adecuadamente los programas de fidelización es relevante para el sector, tanto por el elevado número de empresas que promueven este instrumento de marketing, como por la importancia que los servicios turísticos y de ocio tienen en la oferta de premios que el conjunto de programas (en especial los Multisponsor) proponen a sus afiliados como elemento de dinamización de los mismos (Dekay y otros, 2009). Si bien hay una abundante literatura centrada en la investigación del titular en los programas de fidelización gestionados de forma individualizada (Monosponsor) por empresas de servicios turísticos (Mattila, 2006; Roehm y otros, 2002), poco se conoce sobre el comportamiento del consumidor dentro de estructuras que integran varios sectores empresariales (Multisponsor) (Reinares, 2006).

La estructura de soporte de un programa de fidelización incorpora la práctica totalidad de los elementos y funcionalidades de una estructura de venta directa (Reinares y Ponzoa, 2004) como se detalla a continuación:

- un sistema de control de transacciones de compra (convertibles en puntos) o disfrute de servicios ${ }^{4}$.

- una base de datos o repositorio de información del cliente (en la que registrar los datos personales facilitados por el titular en su alta en el programa, la información transaccional y de interacción con el programa).

- conexiones de la base de datos con los diferentes canales de comunicación directa e interactiva (conjunto de soluciones CRM).

- un sistema capaz de controlar el almacenaje, expedición, transporte y entrega (o recogida por el cliente) de los premios o billetes aéreos.

\footnotetext{
${ }^{3}$ Desde su lanzamiento el programa Aadvantage ha conseguido, según datos publicados por la propia compañía en su informe anual de 2008, más de 62.000.000 de titulares o members. http://www.aa.com

${ }^{4}$ La mayor parte de las aerolíneas acreditan o asignan los puntos o millas al realizar el viaje o conseguir los billetes de embarque, es el caso de los programas Iberia Plus (Iberia), Flying Blue (Air Europa) y Spanair Plus (Spanair) en España , AAdvantage (American Airlines), OnePass (Continental), SkyMilles (Delta) en Estados Unidos o Miles \& More (Lufhansa), Excutive Club (British Airways), Aeroplan (Air Canada), Lan Pass (Lan Chile), Club Premiere (Aero México), Fidelidade (TAM Brasil), Aeropass (Aeropostal) y Phoenix Miles (Air China) en otros mercados.
} 
- un sistema capaz de analizar la información (CRM Analítico) y dinamizar el programa (Gestor de Promociones y Publicidad).

Solo falta, por lo tanto, para completar la plataforma de venta directa (o reconvertir la plataforma de fidelización en una plataforma capaz de ofrecer productos y servicios), añadir una pasarela de pago. Esta funcionalidad puede también estar implantada y experimentada, si el programa ofrece la posibilidad de canje de los premios con puntos más dinero.

\section{LOS PROGRAMAS DE FIDELIZACIÓN Y LAS COMPAÑÍAS AÉREAS}

El desarrollo generalizado de programas de fidelización en este sector es coherente con las aportaciones de la literatura consultada. Para Wansink y Seed (2004) los programas de fidelización funcionan mejor para servicios con márgenes altos, cuya compra se reitera a lo largo del tiempo. Es decir, ofrecen mejores resultados en productos y servicios poco diferenciados, tales como compañías aéreas y hoteles (Nako, 1997).

Estas prácticas empresariales se han reflejado en un gran número de trabajos de investigación centrados en empresas de servicios de transporte aéreo. La antigüedad de los programas en este sector, sus implicaciones económicas, la riqueza de los datos disponibles, o el mayor interés de las compañías por apoyar su gestión con elementos contrastados, explica que gran parte de la investigación aplicada haya elegido este sector. Los programas de las compañías aéreas son analizados desde distintos puntos de vista: su efecto sobre la demanda (Lederman, 2007; Nako, 1997), capacidad para gestionar la heterogeneidad de mercados masivos segmentándolos (Galguera y otros, 2006; Reinares y García de Madariaga, 2008), la tipología de recompensas más adecuada para optimizar los beneficios de los programas (Reinares y Ponzoa, 2008), los efectos de los programas sobre la fidelidad de usuarios en diferentes mercados (García. y otros 2006, Kopalle y Neslin, 2003), y en el sector de las líneas aéreas en particular (Galguera y Méndez, 2004; Gilbert 1996; Mattila, 2006; Liu y otros, 2000) y, en especial, propuestas sobre la forma de gestionar estos programas adecuadamente (Atalik, 2007; Klophaus, 2005; Michels y Bowen 2004).

Las empresas de servicios turísticos que deciden incorporar un programa de fidelización dentro de sus estrategias, pueden plantearse las siguientes opciones:

- Gestionar un programa asociado directamente a su enseña (monosponsor): En esta tipología se encuentran los programas de la mayor parte de las grandes y medianas compañías aéreas, las tarjetas de crédito asociadas al mundo del viaje y, alguno de los grandes grupos de hoteles. En los programas monosponsor, la propiedad de la base de datos y el control del mismo reside en un patrocinador principal (la compañía aérea o grupo hotelero que dota de imagen y contenidos al programa). El propietario puede decidir incluir dentro del programa a otras empresas no competidoras que participan ofreciendo puntos o millas por la contratación de sus respectivos servicios.

- Incorporarse a la estructura de un programa Multisponsor: Los programas Multisponsor surgen del acuerdo entre compañías de diferentes sectores de consumo que deciden ofrecer al titular una oferta amplia de consecución de puntos. A diferencia de los monosponsor, las empresas que potencian o crean un programa Multisponsor depositan en una compañía externa (que suele participar como socio) la propiedad y gestión de la base de datos del programa de fidelización. Es una opción preferida 
por aquellas compañías aéreas que buscan en el programa una forma de intercambiar clientes y ahorro de costes (Klophaus, 2005). Su principal limitación es la pérdida de identificación de la enseña que cede o presta su notoriedad a una marca diferente que la representa en el ámbito de la fidelización.

- Aportar sus servicios como premios del programa: En el caso de ser coincidentes los servicios ofrecidos por los partners con las opciones de premios elegidas por el impulsor del programa 5 . Generalmente los programas Multisponsor incorporan en su oferta premios basados en servicios turísticos por la clara preferencia que sobre los mismos poseen los clientes (ver Gráfico 1 del apartado 7, que recoge los premios canjeados).

\section{IMPORTANCIA DE LAS RECOMPENSAS BASADAS EN SERVICIOS TURÍSTICOS Y DE OCIO}

No percibir como interesantes o adecuadas las recompensas proporcionadas es uno de los principales motivos declarados por el consumidor para no participar o abandonar el programa, según estudios promovidos por sus propios gestores (Reinares y Ponzoa, 2008). El éxito de un programa de fidelización dependerá, en gran medida, de mejorar el valor percibido de las recompensas ofertadas (Meyer-Waarden y Benavent, 2006). Entre las múltiples recompensas ofrecidas por los programas de fidelización, los servicios turísticos ocupan un papel muy destacado. En especial, y como podrá comprobarse en el análisis exploratorio presentado más adelante, son los premios relacionados con viajes en avión y estancias en hoteles, dos de las recompensas más recurrentes, tanto entre programas Monosponsor como Multisponsor.

El alto valor otorgado por el consumidor a la posibilidad de utilizar billetes aéreos de forma gratuita, o hacerlo por un coste muy reducido, es coincidente con la dinámica de los programas en un triple sentido:

- Valor emocional del premio o incentivo: Los programas de fidelización incorporan, entre sus premios, servicios o productos de difícil cuantificación y alto valor percibido por parte del cliente. Se pretende que el titular del programa no logre establecer una relación directa entre esfuerzo realizado (en número de transacciones de compra) y la recompensa (valor en dinero del premio conseguido). Para ello, los gestores de los programas, incluyen premios o servicios en los que el valor real es sustituido por valores emocionales (Mattila, 2006). Dado que el incentivo favorece la participación y la satisfacción con el programa (Meyer-Waarden y Benavent, 2006), el mismo ha de estar en la escala más alta de valoración por parte del titular. El valor emocional del premio ha de servir igualmente a la empresa para conseguir diferenciación con el programa respecto a sus competidores (Yi y Jeon, 2003): encontrar los premios más adecuados incide sobre la participación en el programa (Roehm y otros, 2002).

\footnotetext{
${ }^{5}$ Incluimos dentro de esta clasificación la práctica totalidad de las compañías aéreas incluidas en las grandes alianzas sectoriales internacionales (One World, Star Alliance y Sky Team) en las que el propio programa de fidelización permite al usuario identificar la alianza con puntos o millas convertibles entre todas las compañías participantes. Las tarjetas de crédito especialmente dirigidas a los viajeros frecuentes (Amex, Diners y JCB) y los grandes grupos hoteleros internacionales (Inter-continental, Occidental, Meliá, Tryp, Sol, Concorde y Holiday Inn).
} 
- Recompensa del esfuerzo de participación a través del disfrute compartido del premio: Disfrutar de un vuelo es un acto social que muy frecuentemente se realiza en compañía de otros miembros de la familia o de amistades. Es por ello que prácticamente la totalidad de los programas de fidelización de las compañías aéreas permiten redimir billetes aéreos beneficiándose varias personas con cargo a los puntos de un único asociado. El carácter asociativo, de familia o grupo, refuerza la idea de $c l u b$ o carácter exclusivo del programa (Long y otros, 2003).

- Tiempo de permanencia del socio en el programa: A diferencia de las promociones (Duffy, 1998; Lewis, 2004), los programas de fidelización son diseñados para que los clientes realicen un recorrido amplio en tiempo dentro de los mismos. La limitación temporal de la promoción de ventas favorece la contratación del servicio o compra del producto, al percibir el cliente que existe un coste de oportunidad en no adquirir el producto a tiempo. En los programas de fidelización, el tiempo (traducido en número de compras en distintos establecimientos en el caso de los programas Multisponsor) favorece la consecución de un premio de alto valor percibido, difícilmente accesible con una mera promoción (Ponzoa, 2007). El ajuste entre los factores del margen o porcentaje que la empresa cede al programa por cada compra que el cliente realiza (expresado en la tabla de consecución de puntos), el valor otorgado por el socio a las recompensas propuestas (expresada en la tabla de canje de puntos), y el tiempo necesario que cada segmento o categoría de clientes necesitará para conseguir un premio, son factores clave en el diseño del programa y en su seguimiento por parte de los clientes (Shi y Soman, 2004).

\section{IMPORTANCIA DE LA CLASIFICACIÓN DE CLIENTES PARA LA ADECUACIÓN DEL CATÁLOGO DE PREMIOS}

El programa de fidelización permite obtener una información más detallada y fiable que la que proporcionan las bases de datos convencionales (Rowley, 2005; Cortiñas y otros, 2008). El uso de esta información para el estudio de los perfiles sociodemográficos de los usuarios de los programas de fidelización ha sido el objetivo concreto de trabajos como los de Reinares y García de Madariaga (2008) o Galguera y Méndez (2006). La caracterización de estos segmentos es relevante para una posterior gestión individualizada de los mismos (Allaway y otros, 2006).

La investigación del cliente que facilita y propicia el programa de fidelización (Michels y Bowen, 2004) es especialmente necesaria para la selección de los premios del catálogo que figuran como incentivo en la participación del titular. Distintos estudios muestran que el principal motivo que el consumidor declara para no participar o abandonar el programa es no percibir como interesantes o adecuadas las recompensas proporcionadas por éste (Meyer-Waarden y Benavent, 2006). En consecuencia, caracterizar a sus usuarios en función de sus preferencias de premios es importante, tanto para la selección de premios, como para la adecuación de los mismos a las características de los clientes.

Por otro lado, también es necesario para la adecuada gestión del programa y la optimización de su presupuesto, asegurar una correcta gestión y rotación de los servicios o mercancías almacenadas como incentivos. En este aspecto nuevamente influye de forma decisiva 
el conocimiento de las preferencias del consumidor y el estudio de las series históricas de productos canjeados, pasos previos para llegar a clasificaciones o segmentos de clientes en función del tipo de premio elegido y de la forma en que el mismo es adquirido y disfrutado.

\section{OBJETIVOS}

Como se ha señalando, la participación del titular y los ingresos de la venta directa de servicios desde la estructura del programa de fidelización dependen, en gran medida, de la aceptación que la oferta de premios pueda tener entre las diferentes tipologías de clientes. Por ello es objeto de la presente investigación el establecer una clasificación de clientes en función de sus características socio-demográficas y comportamiento transaccional en el programa, que permita esclarecer la conducta en el canje de billetes aéreos con cargo a puntos y puntos más dinero. Previo a dicha clasificación, y con el objetivo de poner de relevancia el grado de importancia del fenómeno estudiado, es necesario incluir resultados descriptivos, en términos de porcentaje de participación en el canje de billetes aéreos respecto al resto de categorías, y de proporcionalidad del canje realizado con cargo a puntos y puntos más dinero, entre el total de premios conseguidos por parte del titular.

\section{DISEÑO DE LA INVESTIGACIÓN}

La investigación parte del estudio descriptivo de un programa Multisponsor líder en el panorama nacional con una antigüedad de más de trece años y cerca de 1,5 millones de usuarios activos. El perfil de usuario de este programa es:

- un hombre o mujer de clase media y media-alta, residente en capitales de más de cien mil habitantes.

- con un núcleo familiar de uno o dos hijos y un nivel de ingresos de entre treinta mil y sesenta y ocho mil euros anuales.

Se accedió a la base de datos del programa desde la propia gestora del mismo. En el caso del análisis descriptivo (Gráficos 1 y 2 y Tabla 2), se han considerado 11.520 canjes (contenidos en la muestra entregada) realizados en el programa de fidelización para el año 2009. Para la selección de la muestra, en el caso del análisis de clientes que canjean vuelos (árbol de decisión), se consideró que, al menos, el 50\% de los titulares incluidos hubieran canjeado con cargo a puntos más dinero.

También se procuró que la totalidad de los titulares incluidos en los árboles de decisión hubieran realizado al menos un canje en los últimos 10 años, eligiendo, entre los mismos, un total de 150.000 clientes.

En este tipo de modelos inductivos resulta fundamental evaluar cómo se comporta la estructura del árbol para generalizar los datos disponibles en una muestra de datos diferente. Por ello, se seleccionó una muestra de entrenamiento de 50.000 individuos, desde la cual se generaron las reglas, y una muestra de validación de 100.000, en la cual se probó el modelo generado.

Los datos fueron tratados mediante la herramienta Pasw Modeler (de SPSS-IBM). Para la elección del árbol de decisión se experimentó con diferentes opciones: Chaid, Chaid Exhaustivo, C\&RT, QUEST y C15, consiguiéndose el mayor nivel de ajuste (próximo al $68 \%$ ) con el Chaid Exhaustivo (representado en la figura 1). 
Tabla 1

Resumen del modelo utilizado en los árboles de clasificación

\begin{tabular}{|c|c|}
\hline Métodos de crecimiento & C\&RT Exhaustivo para Billetes Aéreos \\
\hline Variables dependientes & Canje con puntos vs. canje con puntos + euros \\
\hline \multirow{12}{*}{$\begin{array}{l}\text { Variables independientes } \\
\text { (T) Transaccional } \\
\text { (SD) Sociodemográfica }\end{array}$} & Sexo (SD) \\
\hline & Edad del titular (SD) \\
\hline & Comunidad autónoma donde reside (SD) \\
\hline & Número de miembros en su familia (SD) \\
\hline & Media de puntos acreditados al mes en el programa de fidelización (T) \\
\hline & Número de años activo en el programa $(\mathrm{T})$ \\
\hline & Autorización expresa para el envío de correo postal (T) \\
\hline & Disponibilidad de correo electrónico (SD) \\
\hline & Autorización para la comunicación vía e-mail (T) \\
\hline & Disponibilidad de teléfono móvil (SD) \\
\hline & Autorización para la comunicación vía sms (T) \\
\hline & Disponibilidad de Internet en domicilio (SD) \\
\hline Validación & SPLITSAMPLE \\
\hline $\begin{array}{l}\text { Casos mínimos en Nodo } \\
\text { Principal }\end{array}$ & 200 \\
\hline $\begin{array}{l}\text { Casos mínimos en Nodo } \\
\text { Secundario }\end{array}$ & 50 \\
\hline Nivel de ajuste & $68 \%$ \\
\hline Tamaño de la muestra total & 150.000 individuos diferentes \\
\hline $\begin{array}{l}\text { Tamaño de la muestra de } \\
\text { validación }\end{array}$ & $\begin{array}{l}100.000 \text { individuos diferentes estratificados al } 50 \% \text { entre quienes } \\
\text { canjean solo con puntos y con puntos más dinero }\end{array}$ \\
\hline
\end{tabular}

\section{RESULTADOS}

\subsection{Participación de la categoría de billetes aéreos respecto a otros tipos de premios (análisis descriptivo)}

En el caso del programa Multisponsor estudiado, el porcentaje de canje de billetes aéreos supone un 10,5\% del total de premios canjeados en unidades (Gráfico 1). Se trata de una participación escasa, si se compara con la categoría más importante: premios tangibles (electrodomésticos, complementos del hogar, bricolaje, cocina...), que son de menor coste unitario y, por lo tanto, de mayor acceso para los titulares que acumulan menos puntos. Sin embargo, si se tiene en cuenta el valor del premio y se realiza una agrupación de los mismos para las categorías estudiadas (Gráfico2), los billetes aéreos suponen un $22 \%$ del total de premios canjeados, un porcentaje significativamente importante y sólo superado por los premios tangibles, dado su importante volumen en unidades (más de un 40\%). 


\section{Gráfico 1}

Porcentaje de canje de billetes aéreos vs. otras categorías de premios en un Programa de Fidelización Multisponsor. Número de unidades (2009)

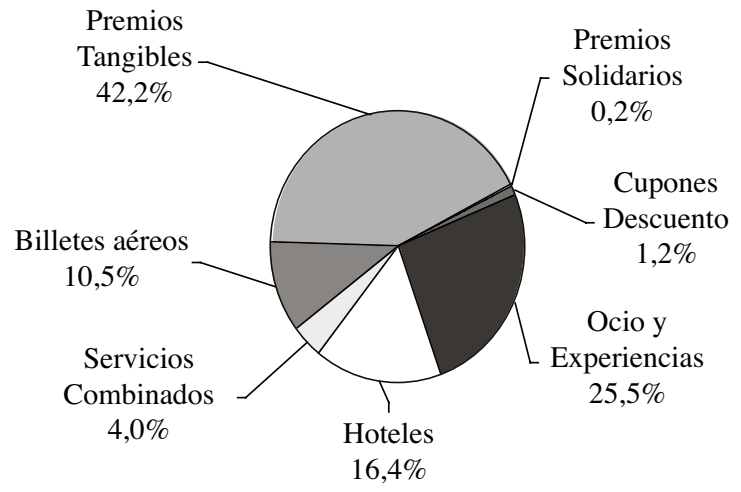

Gráfico 2

Porcentaje de canje de billetes aéreos vs. otras categorías de premios en un Programa de Fidelización Multisponsor. Importe de los premios (2009)

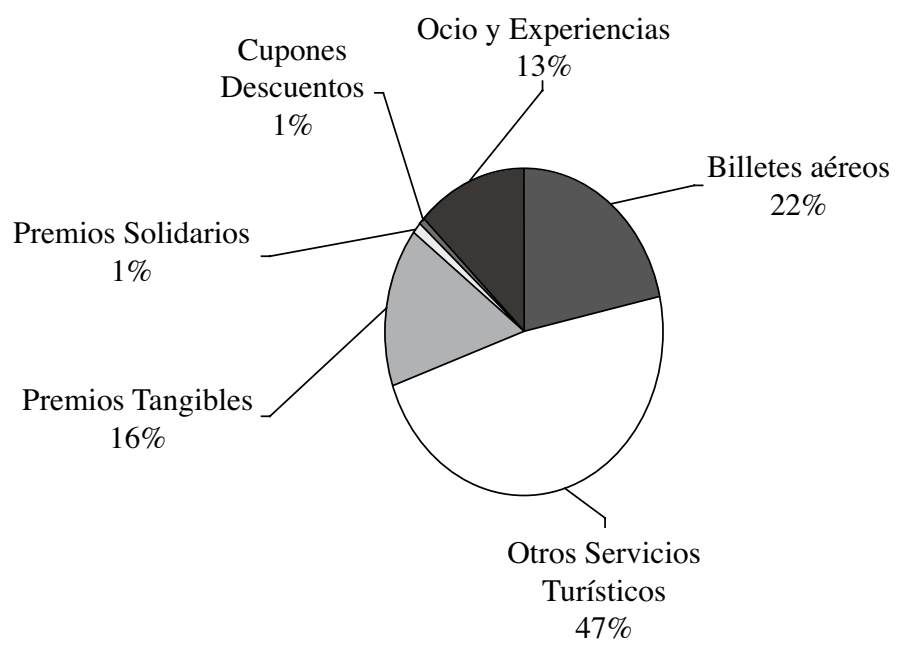

A través del análisis descriptivo se ha podido constatar la importancia de los billetes aéreos dentro de premios ofrecidos en el programa Multisponsor 6 .

\footnotetext{
${ }^{6}$ Equiparable a la oferta de premios existente en la mayor parte de los programas existente tales como Travel Club España, Turyocio España, Travel Club Canada, Néctar U.K. o Travel Club Holanda, entre otros, observable en sus respectivas páginas Web.
} 
En la Tabla 2 se recogen los datos obtenidos del estudio de las series de canje históricas en el programa. Se diferencia dentro de las mismas, y para cada categoría o agrupación de premios: el porcentaje de titulares o socios del programa que canjean, el número total de premios canjeados estimados en unidades de canje, los puntos que los titulares han utilizado en cada caso, y el dinero (euros utilizados) que han añadido los socios del programa para completar el canje o conseguir más unidades o mejores premios.

Es destacable que prácticamente el $95 \%$ de dicho dinero se destina a completar servicios turísticos. Un 30\% de la aportación dineraria pertenece a la financiación de billetes aéreos, siendo, por lo tanto, una categoría objetivo para la venta directa.

Por último, se incluye una distribución o participación del valor de los premios para cada categoría ya comentado.

Tabla 2

Distribución del canje en función de diferentes Parámetros y Tipos de Premios en un Programa Multisponsor (2009)

\begin{tabular}{|l|c|c|c|c|c|}
\hline \multicolumn{1}{|c|}{ Tipo de premio } & Titulares & $\begin{array}{c}\text { Premios } \\
\text { Canjeados }\end{array}$ & $\begin{array}{c}\text { Puntos } \\
\text { Utilizados }\end{array}$ & $\begin{array}{c}\text { Euros } \\
\text { Utilizados }\end{array}$ & $\begin{array}{c}\text { Total Valor } \\
\text { premios }\end{array}$ \\
\hline Billetes aéreos & $15,3 \%$ & $10,5 \%$ & $19,9 \%$ & $29,5 \%$ & $21,7 \%$ \\
\hline Otros Servicios Turísticos & $24,5 \%$ & $20,4 \%$ & $43,5 \%$ & $65,1 \%$ & $47,7 \%$ \\
\hline Premios Tangibles & $42,1 \%$ & $42,2 \%$ & $19,4 \%$ & $1,9 \%$ & $16,0 \%$ \\
\hline Premios Solidarios & $0,4 \%$ & $0,2 \%$ & $1,1 \%$ & $2,1 \%$ & $1,3 \%$ \\
\hline Cupones Descuento & $1,1 \%$ & $1,2 \%$ & $0,6 \%$ & $0,0 \%$ & $0,5 \%$ \\
\hline Ocio y Experiencias & $16,6 \%$ & $25,6 \%$ & $15,5 \%$ & $1,4 \%$ & $12,8 \%$ \\
\hline TOTAL & $100 \%$ & $100 \%$ & $100 \%$ & $100 \%$ & $100 \%$ \\
\hline
\end{tabular}

\subsection{Tipificación del titular que canjea billetes aéreos (modelo probabilístico aplica- do a un programa multisponsor)}

Tal y como se puede comprobar en los resultados incluidos en el árbol mostrado en la Figura 1, el modelo discrimina entre variables transaccionales (puntos acreditados) y variables de índole socio-demográfico (edad y sexo) para realizar la clasificación de titulares. La variable que se sitúa en los nodos iniciales ( 1 y 2) se corresponde con el nivel medio de acreditación del titular, inicialmente 227 puntos mensuales. Éstos son equivalente a compras del titular en las diferentes empresas asociadas al programa de fidelización por un valor aproximado de 550 Euros mensuales. En el segundo nivel del árbol (nodos 3 y 4), es la edad del titular (nacidos antes y después de 1973) la variable que vuelve a mostrarse como relevante en la clasificación de los titulares que canjean con cargo a puntos más euros. La antigüiedad en el programa (nodos 5 y 6) es una de las variables que mejor clasifica a los titulares con menor nivel de acreditación.

Otras características sociodemográficas, como el acceso a Internet, el número de individuos que conviven en el hogar, o la pertenencia a una determinada Comunidad Autónoma (nodos 9 al 18), son también discriminantes. Cada nodo es analizado por separado en la Tabla 3. 
Cabe destacar que los titulares que canjean utilizando dinero son los que acreditan menos puntos (necesitan completar sus puntos para canjear el premio). Su volumen compras asociadas a la tarjeta de fidelidad es de unos $550 €$ de media. Con respecto a los titulares que canjean con sólo puntos, la investigación pone de relevancia que acreditan más puntos de promedio, son mayores de 43 años y se redimen los premios por igual entre hombres y mujeres.

Figura 1

Árbol de decisión (Chaid Exhaustivo) generado para la clasificación de titulares que canjean billetes aéreos en el programa Multisponsor

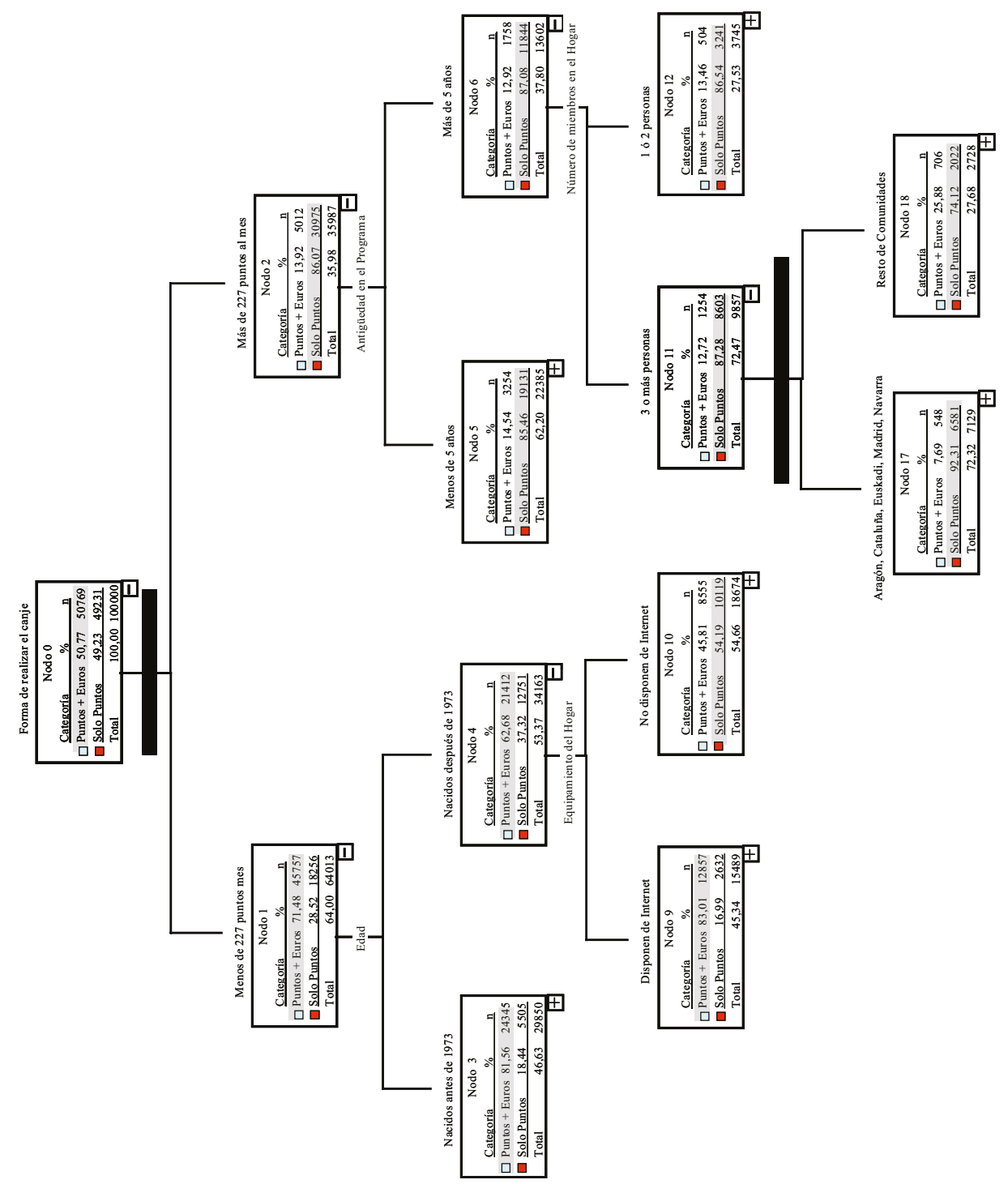


Tabla 3

Principales resultados obtenidos en la clasificación de titulares del programa que canjean billetes aéreos con cargo a puntos y puntos + dinero

\begin{tabular}{|c|c|c|}
\hline NODO & Variable independiente & Resultados \\
\hline 0 & $\begin{array}{l}\text { Forma de realizar el } \\
\text { canje: solo puntos o } \\
\text { puntos+dinero }\end{array}$ & $\begin{array}{l}\text { Muestra estratificada, reparto próximo al 50\% en función del } \\
\text { tipo de canje. }\end{array}$ \\
\hline 1 & $\begin{array}{l}\text { Menos de } 227 \\
\text { puntos acreditados } \\
\text { mes }\end{array}$ & $\begin{array}{l}\text { Son especialmente proclives al canje de vuelos con puntos } \\
+ \text { dinero aquellos titulares que acreditan mensualmente menos de } \\
227 \text { puntos }(64,01 \%) \text { equivalentes a aproximadamente } 550 € \\
\text { de compra asociadas a la tarjeta de fidelidad. }\end{array}$ \\
\hline 2 & $\begin{array}{l}\text { Más de } 227 \text { puntos } \\
\text { acreditados mes }\end{array}$ & $\begin{array}{l}\text { Los clientes que consiguen mayor número de puntos son menos } \\
\text { proclives a completar el canje con dinero. }\end{array}$ \\
\hline 3 & $\begin{array}{l}\text { Nacidos antes de } \\
1973\end{array}$ & $\begin{array}{l}\text { Los titulares con menor ritmo de acreditación de } \\
\text { puntos canjean preferentemente con puntos + dinero cuando su } \\
\text { edad es inferior a } 37 \text { años. }\end{array}$ \\
\hline 4 & $\begin{array}{l}\text { Nacidos después de } \\
1973\end{array}$ & $\begin{array}{l}\text { En el caso de los titulares con menor ritmo de acreditación, } \\
\text { mayores de } 37 \text { años, existe correlación entre quienes disponen de } \\
\text { acceso a Internet (nodos } 9 \text { y 10) y canjean con puntos + dinero. }\end{array}$ \\
\hline 5 & $\begin{array}{l}\text { Menos de } 5 \text { años } \\
\text { de antigüedad en el } \\
\text { Programa }\end{array}$ & $\begin{array}{l}\text { Los titulares que acreditan más número promedio de puntos } \\
\text { mensuales canjean en su mayor parte vuelos pagando solo en } \\
\text { puntos cuando tienen una antigüedad en el programa menor de } \\
5 \text { años. }\end{array}$ \\
\hline 6 & $\begin{array}{l}\text { Más de } 5 \text { años de } \\
\text { antigüedad en el } \\
\text { Programa }\end{array}$ & $\begin{array}{l}\text { Los titulares que más tiempo llevan en el programa y más puntos } \\
\text { acreditan, disponen frecuentemente de la totalidad de puntos } \\
\text { necesarios para canjear un vuelo, por lo que no suelen utilizan } \\
\text { dinero para completar el canje. }\end{array}$ \\
\hline 9 & $\begin{array}{l}\text { Disponen de } \\
\text { Internet }\end{array}$ & $\begin{array}{l}\text { Existe mayor probabilidad de pagar con cargo a puntos + dinero } \\
\text { entre aquellos titulares habituados a utilizar Internet. }\end{array}$ \\
\hline 10 & $\begin{array}{l}\text { No disponen de } \\
\text { Internet }\end{array}$ & $\begin{array}{l}\text { Al igual que ocurre en el nodo } 9 \text {, pero en este caso con signo } \\
\text { contrario, las personas menos habituadas a las nuevas tecnolo- } \\
\text { gías son las que más reticentes se muestran a utilizar dinero para } \\
\text { completar el canje de billetes de avión. }\end{array}$ \\
\hline 11 & $\begin{array}{l}3 \text { o más personas en } \\
\text { el hogar }\end{array}$ & $\begin{array}{l}\text { En el caso de los titulares con más ritmo de acreditación y más } \\
\text { años de pertenencia al programa, la variable número de miem- } \\
\text { bros en el hogar es relevante en la clasificación de aquellos que } \\
\text { canjean con cargo a puntos + euros. }\end{array}$ \\
\hline 12 & $\begin{array}{l}1 \text { ó } 2 \text { personas en el } \\
\text { hogar }\end{array}$ & $\begin{array}{l}\text { Los resultados del nodo } 12 \text { vienen a confirmar lo señalado en el } \\
\text { nodo } 11 \text {, aunque los titulares viajen solos o lo hagan en pareja, } \\
\text { aquellos que tienen una mayor implicación con el programa (en } \\
\text { tiempo y número de transacciones de compras asociadas al mis- } \\
\text { mo) tienen menor necesidad de completar sus vuelos con dinero } \\
\text { al disponer de mayor número de puntos disponibles. }\end{array}$ \\
\hline
\end{tabular}




\begin{tabular}{|c|l|l|}
\hline NODO & Variable independiente & \multicolumn{1}{c|}{ Resultados } \\
\hline 17 & $\begin{array}{l}\text { CCAA: Aragón, } \\
\text { Cataluña, Euskadi, } \\
\text { Madrid, Navarra }\end{array}$ & $\begin{array}{l}\text { Son las comunidades autónomas con mayor renta per cápita y } \\
\text { densidad de población (en el caso de Madrid y Cataluña) aque- } \\
\text { llas en las que residen los titulares que, teniendo más afinidad } \\
\text { al programa y número de miembros en el hogar deciden canjear } \\
\text { con cargo a puntos. }\end{array}$ \\
\hline 18 & $\begin{array}{l}\text { Resto de } \\
\text { Comunidades }\end{array}$ & $\begin{array}{l}\text { La afirmación realizada para el nodo 17 es también cierta para } \\
\text { el caso del resto de comunidades, si bien, en dicho caso la dife- } \\
\text { rencia entre aquellos que canjean y no utilizando el dinero para } \\
\text { completar su premio no es tan importante, solo un 25,9 \% de los } \\
\text { titulares utilizan dinero para completar el canje. }\end{array}$ \\
\hline
\end{tabular}

\section{CONCLUSIONES}

Los billetes aéreos y, en general, los premios relacionados con el sector turístico, son un premio de alto nivel de aceptación entre los usuarios de los programas de fidelización. Esta característica es más evidente cuando el programa incluye a usuarios capaces de conseguir los puntos necesarios para sufragar el premio, es decir, cuando el comportamiento de compra (reiteración de transacciones en los patrocinadores que ofrecen puntos) y la capacidad de consumo (importe gastado en las empresas asociadas al programa), permiten a los titulares obtener puntos suficientes para conseguir este tipo de premios de mayor valor promedio frente al resto de premios incluidos como incentivos. El 39,8 \% de los titulares canjean el 69,4\% del valor de los premios asociados a servicios turísticos en un programa Multisponsor.

Si bien el número de puntos disponibles es determinante en la decisión de sufragar un billete aéreo añadiendo dinero, también resultan relevantes variables socio-demográficas como la edad y el acceso a Internet.

Se confirma, por lo tanto, la tendencia presentada en algunos de los últimos informes publicados en España sobre comercio electrónico y venta directa e interactiva ${ }^{7}$. Se puede observar como, entre otras causas, la venta directa al consumidor crece en la medida que se incorporan al mercado consumidores jóvenes que van disponiendo paulatinamente de mayor nivel adquisitivo. Dichos compradores creen en la potencialidad de los canales de comunicación directa e interactiva como vía para el ahorro de costes de intermediación y acceso rápido y cómodo (sin desplazamiento) a la compra.

La estructura de soporte utilizadas para la gestión y administración de los programas de fidelización, y la propia dinámica o reglas de participación en el programa, permiten el canje de premios con cargo a puntos más dinero. Como se señalaba anteriormente, existen muy pocas diferencias entre la estructura de venta directa y la de un programa de fidelización. La primera permite cambiar dinero por bienes o servicios y la segunda permite el

\footnotetext{
7 Tales como el "Estudio Global de Marketing Directo e Interactivo" (2008) presentado por Correos son la colaboración de ESIC , el "Informe del Comercio Electrónico en España a través de entidades de Medios de Pago" (2009) elaborado por la Comisión del Mercado de las Telecomunicaciones o el "Informe sobre Comercio Electrónico B2C en España" (2008) del Observatorio Nacional de las Telecomunicaciones y de la Sociedad de la Información (ONTSI) de Red.es, entidad del Ministerio de Industria, Turismo y Comercio.
} 
intercambio de puntos (u otra moneda propia) por premios (entre los que pueden incluir bienes tangibles, viajes, vuelos u otro tipo de servicios).

A la facilidad de una estructura ya existente para la venta directa, hay que añadir otros factores de éxito, tales como la confianza que otorga o genera en el comprador el propio programa de fidelización (titular del programa durante un largo periodo de tiempo), o la posibilidad de añadir ofertas sin que la compañía aérea vea afectada su política de precios al ocultar su valor.

Existiendo un sistema de soporte (tecnológico y humano), negocio (derivado de la frecuencia en la utilización por parte del titular del servicio de pago con dinero de parte del premio), y conocimiento del producto (derivado de las campañas comerciales que frecuentemente utilizan el programa para incentivar la oferta), todo hace pensar que los programas de fidelización están experimentando una evolución para convertirse también en plataformas de venta directa. De hecho, dichas plataformas relacionales están sirviendo, especialmente en las compañías aéreas, como vía para la entrada de clientes con los que establecer un trato personal y directo basado en la venta y recomendación a terceras personas (muy probablemente afines a los gustos, preferencias y comportamiento en la compra de quien recomienda). Dichas plataformas de fidelización y venta directa son interesantes para las empresas promotoras de plataformas Multisponsor (en el sentido de creación de nuevas unidades de negocio), para las plataformas Monosponsor (que pueden comercializar sus propios productos o servicios), y para los operadores turísticos asociados (que pueden comercializar vuelos u otros servicios siendo proveedores de las mismas). No es por lo tanto casual, que una importante nómina de empresas del sector, participen en uno o varios programas de fidelización (Monosponsor o Multisponsor), y lo hagan, además, contribuyendo con recompensas (puntos) y como proveedoras de vuelos y otros servicios turísticos.

Desde las perspectiva de las implicaciones del estudio para la gestión de la empresa, tanto los resultados referidos a los premios conseguidos con puntos y con puntos y dinero (a la postre facturación para el proveedor del servicio), como las clasificaciones de clientes que canjean facilitadas por el árbol de decisión, pueden ser utilizadas como referencia para aquellas empresas que gestionan o están pensando desarrollar un programa de fidelización. En este sentido podría reducirse la incertidumbre en la toma de decisiones si se pudiera realizar un análisis, fundamentado en el modelo propuesto, sobre la propia base de datos de clientes de la compañía aérea interesada en participar en el programa, con el objeto de establecer correlaciones entre los clientes y los titulares participantes. De igual forma, los resultados obtenidos, aportan información que puede ser utilizada para estimular la participación de los titulares en al programa al ajustar de forma más precisa los beneficios. Para concluir, se ha contrastado la importancia del CRM Analítico para estudiar las preferencias de los consumidores en los programas de fidelización.

\section{FUTURAS LÍNEAS DE INVESTIGACIÓN}

Una de las principales limitaciones del estudio se deriva de la amplia clasificación de los servicios turísticos, mientras que el presente trabajo limita su enfoque a las compañías aéreas. Si bien se considera que las implicaciones de este trabajo pueden ser validas, además de para las compañías áreas, para otras empresas de servicios turísticos que pro- 
mueven programas de fidelización, se propone, para una futura investigación, la repetición del estudio considerando otras categorías de servicios, tales como hoteles y sus tipologías (ciudad playa, categoría en estrellas, de destinos nacionales o internacionales), tipologías de operadores (agencias on y off line, mayoristas, proveedores de viajes, otros intermediarios) o servicios combinados por destinos y tipo.

En lo referido a la contratación de transportes para desplazamiento de pasajeros, apreciamos igualmente una limitación en el estudio el considerar únicamente el transporte aéreo. En este sentido, sería revelador para dicha categoría, incluir nuevas opciones tales como el alquiler de vehículos y el transporte en autobús, $\operatorname{tren}^{8}$ o barco.

\section{REFERENCIAS BIBLIOGRÁFICAS}

ALLAWAY, A. W.; GOONER, R. M.; BERKOWITZ, D. Y DAVIS, L. (2006): "Deriving and exploring behaviour segments within a retail loyalty card program", European Journal of Marketing, Vol. 40, $\mathrm{n}^{\circ} 11 / 12$, pp. 1317-1339.

ATALIK, Ö. (2007): "Customer complaints about airline service: a preliminary study of Turkish frequent flyers", Management Research News, Vol. 30, nº 6, pp. 409-419.

BANASIEWICZ, A. (2005): "Loyalty program planning and analytics", Journal of Customer Marketing, Vol. 22, n6; pp.332-339.

CARLSSON, F. Y LÖFGREN, A. (2006): "Airline choice, switching costs and frequent flyer programmes", Applied Economics; Vol.38; n 13; pp. 1469-1475.

CORTIÑAS, M., ELORZ, M. Y MÚGICA J.M. (2008): “The use of loaylty-card databases: differences in regular price and discount sensitivity in the brand choice decision between card and non-card holders", Journal of Retailing and Consumer Services; Vol.15, n 1; Págs 52-62.

CORREOS- ESIC (2008): "I Estudio Global de Marketing Directo e Interactivo", Publicaciones de Correos, Madrid.

CMT (2009): Informe del Comercio Electrónico en España a través de entidades de Medios de Pago, Comisión del Mercado de las Telecomunicaciones, Barcelona.

DEKAY, F. TOH, R. S. Y RAVEN, P. (2009): “Loyalty Programs: Airlines Outdo Hotels”, Cornell Hospitality Quarterly, Vol. 50, nº. 3, pp. 371-382.

DUFFY, D. (1998): “Customer loyalty strategies”, Journal of Consumer Marketing, Vol 15, nº.1, pp. 435-448.

GALGUERA, L. Y MÉNDEZ, M. P. (2004): "Una aproximación empírica al marketing de relaciones. Las tarjetas de fidelización de las compañías aéreas”, Esic Market, septiembre-diciembre, n'.119, pp. 223-256.

GALGUERA, L.; LUNA, L.; LUNA, D Y MÉNDEZ, M. P. (2006): "Predictive segmentation in action. Using CHAID to segment loyalty cardholders", International Journal of Market Research, Vol. 48, n. 4, pp. 459-479.

GARCÍA, B. GUITIÉRREZ, A.M. Y GUTIÉRREZ, J.(2006): "The role of loyalty programs in behavioral and affective loyalty”. Journal of Consumer Marketing, Vol.23; no 7; pp. 387-396.

GILBERT, D.C.(1996): "Relationship marketing and airlines loyalty schemes", Tourism Management; Vol.17, $\mathrm{n}^{\mathrm{o}} 8$, pp. 575-582.

\footnotetext{
${ }^{8}$ Según puede desprenderse del Barómetro OMT de Turismo Mundial de Enero de 2009, Vol. 7 n 1 . España 2009, la venta de billetes aéreos se está desplazando hacia los trenes de alta velocidad, dicha peculiaridad podría ser también investigada desde la perspectiva del perfil de clientes que está protagonizando dicho cambio.
} 
KLOPHAUS, R. (2005): "Frequent flyer programs for European low-cost airlines: Prospects, risks and implementation guidelines", Journal of Air Transport Management, Vol. 11, n 5, pp. 348-353.

KOPALLE P.K. Y NESLIN, S.A. (2003): "The economic viability of frequency reward programs in a strategic competitive environment", Review of Marketing Science; Vol.1, $\mathrm{n}^{\circ} 1$; pp. 1-39

LEDERMAN, M. (2007): "Do enhancements to loyalty programs affect demand? The impact of international frequent flyer partnerships on domestic airline demand", Journal of Marketing Research, Vol. 38, no 4, pp. 1134-1158.

LEENHEER, J.; VAN HEERDE, H. J.; BIJMOLT, T. H. A. Y SMIDTS, A. (2007): "Do loyalty programs really enhance behavioural loyalty? An empirical analysis accounting for self-selecting members", International Journal of Research in Marketing, Vol. 24, nº 1, pp. 31-47.

LEWIS, M. (2004): "The influence of loyalty programs and short-term promotions on customer retention". Journal of Marketing Research, Vol. 41, n³, pp. 281-292.

LIU, A., WALL, G Y WESTLAKE, J.(2000): "Marketing through frequent flyer programs: the example of China Airlines", Tourism Economics, Vol.6; n 3, pp.233-249.

LONG, M.M; CLARK, S.D. SCHIFFMAN, L.G. Y MCMELLON, C. (2003): "In the air again: frequent flyer relationship programmes and business travellers' quality of life", International Journal of Tourism Research, Vol. 5, N.6, pp. 422-432.

MATTILA, A. (2006): "How Affective Commitment Boosts Guest Loyalty (and Promotes Frequent-guest Programs)”, Cornell Hotel and Restaurant Administration Quarterly, Vol. 47, n² pp. 174-181.

MEYER-WAARDEN, L. Y BENAVENT C. (2006): "La Théorie de la Motivation Extrinsèque et Intrinsèque Appliquée a la Problématique des Gratifications de Programmes de Fidélisation", Proceedings AMF French Marketing Academy, Nantes, pp. 1-44.

MICHELS,N Y BOWEN,D. (2004): "The relevance of retail loyalty strategy and practice for leisure/tourism", Journal of Vacation Marketing, Vol. 11, no 1, pp. 5-19.

NAKO, S. (1997): "Frequent flyer programs and business travelers: An empirical investigation". Logistics and Transportation Review, Vol. 28, nº, pp. 395-410.

OMT (2009): "Barómetro OMT de Turismo Mundial", OMT, Enero, Vol.7, nº1, España.

PONZOA, J.M. (2007): Programas de fidelización de clientes: un estudio empírico sobre la efectividad de los canales de comunicación directa e interactiva en la dinamización de ventas y relaciones, Tesis Doctoral, Universidad Complutense de Madrid, Madrid.

REINARES, P. (2006): "Exploring the benefits obtained in a Loyalty Program applied to Retailing", European Retail Digest, Vol. 48. pp. 48-52.

REINARES, P. Y GARCÍA DE MADARIAGA, J. (2008): "The importance of rewards in the management of Multisponsor loyalty programmes", Journal of Database Marketing \& Customer Strategy Management, Vol.15, nº 1 , pp. 37-48.

REINARES, P. Y PONZOA, J.M. (2008): "Improving management of multi-sponsor loyalty programmes based on the rewards preferred by consumers", Journal of Customer Behaviour, Vol.7, n'2, pp. 121-134.

REINARES, P. Y PONZOA, J.M. (2004): Marketing Relacional, Prentice-Hall Financial Times, Madrid

RED.ES (2008): Informe sobre Comercio Electrónico B2C en España, Observatorio Nacional de las Telecomunicaciones y de la Sociedad de la Información (ONTSI), Ministerio de Industria, Turismo y Comercio.

ROEHM, M. L.; BOLMAN PULLINS, E. Y ROEHM JR, H.A. (2002): “Designing Loyalty-Building Programs for Packaged Goods Brands", Journal of Marketing Research, Vol. 39, pp. 202-213.

ROWLEY, J. (2005): "Building brand webs: Customer relationship management through the Tesco Clubcard loyalty scheme", International Journal of Retail \& Distribution Management, Vol. 33, $\mathrm{n}^{\circ} 3$, pp. $194-206$. 
SHI, M. Y SOMAN, D. (2004): Multi-Medium Reward Programs, Working Paper, Rotman School, Toronto.

VÁZQUEZ, R., DÍAZ, A.M. Y SUÁREZ, A. (2004) "Cómo usan Internet las líneas aéreas para desarrollar relaciones estables con los clientes", Universia Business Review, Segundo trimestre, $\mathrm{n}^{\circ} 002$, pp.34-47.

WANSINK, B. Y SEED, S. (2001): "Making Brand Loyalty Programs Succeed", Journal of Brand Management, Vol. 8, $\mathrm{n}^{\circ}$ 2, pp. 211-222.

YI, Y. Y JEON, H. (2003): "Effects of loyalty Programs on Value Perception, Program loyalty, and Brand loyalty”, Journal of the Academy of Marketing Science, Vol. 31, n³, pp. 229-240. 
